\title{
Dynamic Response of Structures With Frequency Dependent Damping Models
}

\author{
Blanca Pascual* and Sondipon Adhikari ${ }^{\dagger}$ \\ Swansea University, Swansea, United Kingdom
}

\begin{abstract}
The calculation of dynamic response of multiple-degree-of-freedom linear systems with frequency dependent damping is considered. It is assumed that the damping forces depend on the past history of velocities via convolution integrals over exponentially decaying kernel functions. Exact closed-form expressions for the dynamic response due to general forces and initial conditions are derived in terms of the eigensolutions of the system in the original space. Eigensolutions of the damped system in turn are obtained approximately as functions of the undamped eigensolutions. This enables one to approximately calculate the dynamic response of complex systems with frequency dependent damping by simple post-processing of the undamped eigensolutions. Suitable examples are given to illustrate the derived results.
\end{abstract}

\section{Introduction}

$\mathrm{T}$ HE characterization of energy dissipation or damping in complex vibrating structures such as aircrafts and helicopters is of fundamental importance. Noise and vibration are not only uncomfortable to the users of these complex dynamical systems, but also may lead to fatigue, fracture and even failure of such systems. Increasing use of composite structural materials, active control and health-monitoring systems in the industry requires the development of new reliable damping models and corresponding computational methods. This paper is aimed at developing computationally efficient and physically insightful approximate numerical methods for linear dynamical systems with frequency dependent damping. Frequency depended or non-viscous/viscoelastic damping can be incorporated in various ways. Here we use the Biot model ${ }^{1}$ (also known as the exponential model) which allows one to incorporate wide range of functions in the frequency domain by means of summation of simple 'pole residue forms'. Several authors have considered this model due to its simplicity and generality.

Viscous damping is by far the most common model for modelling of vibration damping in linear systems. It used widely for their simplicity and mathematical convenience even though the behavior of real structural materials is, at best, poorly mimicked by simple viscous models. It is well recognized that in general a physically realistic model of damping will not be viscous. Damping models in which the dissipative forces depend on any quantity other than the instantaneous generalized velocities are non-viscous damping models. Mathematically, any causal model which makes the energy dissipation functional non-negative is a possible candidate for a non-viscous damping model. Possibly the most general way to model damping within the linear range is to use non-viscous damping models which depend on the past history of motion via convolution integrals over kernel functions. The equations of motion of a $N$-degree-of-freedom linear system with such damping can be expressed by

$$
\mathbf{M} \ddot{\mathbf{u}}(t)+\int_{0}^{t} \mathcal{G}(t-\tau) \dot{\mathbf{u}}(\tau) \mathrm{d} \tau+\mathbf{K u}(t)=\mathbf{f}(t)
$$

${ }^{*}$ Graduate Student, School of Engineering, Swansea University, Singleton Park, Swansea SA2 8PP, UK.

${ }^{\dagger}$ Professor of Aerospace Engineering, School of Engineering, Swansea University] Singleton Park, Swansea SA2 8PP, UK, AIAA Senior Member.

Copyright (c) 2008 by the American Institute of Aeronautics and Astronautics, Inc. The U.S. Government has a royalty-free license to exercise all rights under the copyright claimed herein for Governmental purposes. All other rights are reserved by the copyright owner. 
together with the initial conditions

$$
\mathbf{u}(t=0)=\mathbf{u}_{\mathbf{0}} \in \mathbb{R}^{N} \quad \text { and } \quad \dot{\mathbf{u}}(t=0)=\dot{\mathbf{u}}_{\mathbf{0}} \in \mathbb{R}^{N} .
$$

Here $\mathbf{u}(t) \in \mathbb{R}^{N}$ is the displacement vector, $\mathbf{f}(t) \in \mathbb{R}^{N}$ is the forcing vector, $\mathbf{M} \in \mathbb{R}^{n}$ is the mass matrix, $\mathbf{K} \in \mathbb{R}^{n}$ is the stiffness matrix and $\mathcal{G}(t-\tau)$ is the matrix of damping kernel functions. The kernel functions $\mathcal{G}(t-\tau)$ are known as retardation functions, heredity functions, after-effect functions or relaxation functions in the context of different subjects. In the limit when $\mathcal{G}(t-\tau)=\mathbf{C} \delta(t-\tau)$, where $\delta(t)$ is the Dirac-delta function, equation (1) reduces to the case of viscous damping.

Equation (1) is very general and for any engineering applications some specific form of $\mathcal{G}(t)$ have to be assumed. A wide variety of mathematical expressions could be used for the kernel functions $\mathcal{G}(t)$ as long as the rate of energy dissipation is non-negative. Some of the damping functions used in the literature are shown in Table 1. A well known damping model, known as the fractional derivative model, ${ }^{3,9-11}$ appears as

Table 1. Some non-viscous damping functions in the Laplace domain

\begin{tabular}{|c|c|c|}
\hline $\begin{array}{l}\text { Model } \\
\text { Number }\end{array}$ & Damping function & Author and year of publication \\
\hline 1 & $G(s)=\sum_{k=1}^{n} \frac{a_{k} s}{s+b_{k}}$ & $\operatorname{Biot}^{2}-1955$ \\
\hline 2 & $G(s)=\frac{E_{1} s^{\alpha}-E_{0} b s^{\beta}}{1+b s^{\beta}} \quad(0<\alpha, \beta<1)$ & Bagley and Torvik ${ }^{3}-1983$ \\
\hline 3 & $s G(s)=G^{\infty}\left[1+\sum_{k} \alpha_{k} \frac{s^{2}+2 \xi_{k} \omega_{k} s}{s^{2}+2 \xi_{k} \omega_{k} s+\omega_{k}^{2}}\right]$ & $\begin{array}{l}\text { Golla and Hughes }{ }^{4}-1985 \\
\text { and McTavish and Hughes }{ }^{5}-1993\end{array}$ \\
\hline 4 & $G(s)=1+\sum_{k=1}^{n} \frac{\Delta_{k} s}{s+\beta_{k}}$ & Lesieutre and Mingori ${ }^{6}-1990$ \\
\hline 5 & $G(s)=c \frac{1-e^{-s t_{0}}}{s t_{0}}$ & Adhikari $^{7}-1998$ \\
\hline 6 & $G(s)=\frac{c}{s t_{0}} \frac{1+2\left(s t_{0} / \pi\right)^{2}-e^{-s t_{0}}}{1+2\left(s t_{0} / \pi\right)^{2}}$ & Adhikari $^{7}-1998$ \\
\hline 7 & $G(s)=c e^{s^{2} / 4 \mu}\left[1-\operatorname{erf}\left(\frac{s}{2 \sqrt{\mu}}\right)\right]$ & Adhikari and Woodhouse ${ }^{8}-2001$ \\
\hline
\end{tabular}

a specific case when the Laplace transform of $\mathcal{G}(t)$ is selected as $s \mathbf{G}(s)=\sum_{j} s^{\nu_{j}} \mathbf{g}_{j}$ where $\mathbf{g}_{j}$ are complex constant matrices and $\nu_{j}$ are fractional powers. Here we will use a damping model for which the kernel function matrix has the special form

$$
\mathcal{G}(t)=\sum_{k=1}^{n} \mu_{k} e^{-\mu_{k} t} \mathbf{C}_{k}
$$

or in the Laplace domain

$$
\mathbf{G}(s)=\sum_{k=1}^{n} \frac{\mu_{k}}{s+\mu_{k}} \mathbf{C}_{k} .
$$

The constants $\mu_{k} \in \mathbb{R}^{+}$are known as the relaxation parameters and $n$ denotes the number relaxation parameters used to describe the damping behavior. This model will also be refereed as the exponential damping model (models 1,3 and 4 in Table 1) for the obvious reason. From equation (3) observe that in the limit when $\mu_{k} \rightarrow \infty, \forall k$ the exponential model reduces to the viscous damping model with an equivalent viscous damping matrix

$$
\mathbf{C}=\sum_{k=1}^{n} \mathbf{C}_{k} .
$$

A physical justification (using the principles of mechanics and thermodynamics) as to why a general structure should always have this type of damping is hard to provide. However, based on engineering judgement and intuition, several reasons behind the selection of this model could be given: 
- In the context of viscoelastic materials, the physical basis for exponential models has been well established - as in the words of Cremer and Heckl: ${ }^{12}$ 'Of the many after-effect functions that are possible in principle, only one — the so-called relaxation function — is physically meaningful.'

- In a large engineering structure it is possible to have different damping in different parts of a structure. For example, various members of a space-frame may have different damping properties, each characterized by a relaxation parameter $\mu_{k}$. Then the associated coefficient matrix $\mathbf{C}_{k}$ would have non-zero blocks corresponding to the relevant elements only. One could perform experiments for individual members and use the finite element method to obtain the element damping matrix, say $\mathbf{C}_{k}^{(e)}$. Using standard approach it is possible to assemble all the element matrices associated with relaxation parameter $\mu_{k}$ to obtain a global damping matrix $\mathbf{C}_{k}$. This procedure may be repeated for all damping types present in the structure to obtain $\mu_{k}$ and $\mathbf{C}_{k}$ for all $k$.

- A mathematical rationalization of this model can be given in terms of the Laplace transform of $\mathcal{G}(t)$. The matrix $\mathbf{G}(s)$ in equation (4) is in general a matrix of complex functions. From the theory of complex variables it is well known that a wide range of complex functions can be represented in the 'pole-residue' form. From equation (4) it is easy to see that $\lambda_{k}$ and $\mathbf{C}_{k}$ are directly related to the poles and residues of $\mathbf{G}(s)$. Therefore, many damping models (except the fractional derivative model which has branch-points due to the fractional powers) can be essentially be represented in the form of (3).

Equation (1) together with the kernel in Eq. (3) represent a set of coupled integro-differential equations. Several authors have proposed ${ }^{3-6,13-17}$ state-space approach based on the internal variables for this type of equations.

In spite of attractive and general damping modeling capabilities, frequency depended non-viscous damping models are not widely used in engineering practice. Most of the vibration analysis textbooks, commercial finite element packages and experimental modal analysis software only allow viscous damping. The main reasons for not using a frequency dependent non-viscous damping model include, but not limited to:

- although exact in nature, the state-space approach usually needed for this type of damped systems is computationally very intensive for real-life systems;

- the physical insights offered by methods in the original space (eg, the modal analysis) is lost in a state-space based approach

- the experimental identification of the parameters of a frequency dependent damping model is difficult.

Regarding the first point, Woodhouse ${ }^{18}$ and Adhikari ${ }^{19}$ proposed approximate methods in the space of the original problem. These methods are applicable when the damping is small and they neglect the overdamped modes. A direct time-domain approach to obtain the solution of Eq. (1) was proposed by Adhikari and Wagner. ${ }^{20}$ This method is computationally efficient and accurate but does not provide much physical insight. Regarding the second point, in general, a linear system with frequency dependent non-viscous damping is expected to have complex modes. ${ }^{21,22}$ The physical justification of complex modes obtained directly from the state-space analysis is still not clear. Regarding the third point, there is no system identification method yet proposed to obtain the relaxation parameters and the associated coefficient matrices in Eq. (4) from vibration measurement. Only for the very special case when $n=1$, Adhikari and Woodhouse ${ }^{23,24}$ proposed an identification method based on complex modal analysis. In this paper we aim to address the first two issues.

Dynamic response of frequency-dependent damped systems can be obtained exactly in closed-form using the eigensolutions of the system. ${ }^{19}$ Therefore, the main challenge is in obtaining the eigensolutions which is the main theme of this paper. The eigenvalue problem corresponding to the equation of motion (1) is in general a non-linear eigenvalue problem. We have derived closed-form approximate expressions of the eigenvalues and eigenvectors of the system for four mathematically different cases based on the values of (a) number of degrees of freedom, and (b) number of kernel functions. The approximations utilize Taylor series expansion in the complex domain and are based on certain simplifying assumptions. The validity of the assumptions and the accuracy of the results are verified by numerical calculations. 


\section{Dynamic Response for the General Case}

Taking the Laplace transform of equation (1) and considering the initial conditions in (2) we have

$$
\begin{aligned}
& s^{2} \mathbf{M} \overline{\mathbf{q}}-s \mathbf{M} \mathbf{q}_{0}-\mathbf{M} \dot{\mathbf{q}}_{0}+s \mathbf{G}(s) \overline{\mathbf{q}}-\mathbf{G}(s) \mathbf{q}_{0}+\mathbf{K} \overline{\mathbf{q}}=\overline{\mathbf{f}}(s) \\
\text { or } \quad & \mathbf{D}(s) \overline{\mathbf{q}}=\overline{\mathbf{f}}(s)+\mathbf{M} \dot{\mathbf{q}}_{0}+[s \mathbf{M}+\mathbf{G}(s)] \mathbf{q}_{\mathbf{0}} .
\end{aligned}
$$

Here the dynamic stiffness matrix is defined as

$$
\mathbf{D}(s)=s^{2} \mathbf{M}+s \mathbf{G}(s)+\mathbf{K} \in \mathbb{C}^{n} .
$$

The inverse of the dynamics stiffness matrix, known as the transfer function matrix, is given by

$$
\mathbf{H}(s)=\mathbf{D}^{-1}(s) \in \mathbb{C}^{n} .
$$

Using the residue-calculus the transfer function matrix can be expressed like a viscously damped system as

$$
\mathbf{H}(s)=\sum_{j=1}^{m} \frac{\mathbf{R}_{j}}{s-s_{j}} .
$$

where $m$ is the number of non-zero eigenvalues (order) of the system. The residue matrices can be expressed as (see Appendix A for details)

$$
\mathbf{R}_{j}=\frac{\mathbf{z}_{j} \mathbf{z}_{j}^{T}}{\mathbf{z}_{j}^{T} \frac{\partial \mathbf{D}\left(s_{j}\right)}{\partial s_{j}} \mathbf{z}_{j}} .
$$

Here $s_{j}$ and $\mathbf{z}_{j}$ are respectively the eigenvalues and eigenvectors of the system, which are solutions of the non-linear eigenvalue problem

$$
\mathbf{D}\left(s_{j}\right) \mathbf{z}_{j}=\mathbf{0}, \quad \text { for } j=1, \cdots, m
$$

where

$$
\mathbf{D}\left(s_{j}\right)=s_{j}^{2} \mathbf{M}+s_{j} \mathbf{G}\left(s_{j}\right)+\mathbf{K} .
$$

This expression of $\mathbf{H}(s)$ in (9) allows the response to be expressed as modal summation as

$$
\overline{\mathbf{q}}(s)=\sum_{j=1}^{m} \gamma_{j} \frac{\mathbf{z}_{j}^{T} \overline{\mathbf{f}}(s)+\mathbf{z}_{j}^{T} \mathbf{M} \dot{\mathbf{q}}_{\mathbf{0}}+s \mathbf{z}_{j}^{T} \mathbf{M} \mathbf{q}_{\mathbf{o}}+\mathbf{z}_{j}^{T} \mathbf{G}(s) \mathbf{q}_{\mathbf{0}}(s)}{s-s_{j}} \mathbf{z}_{j}
$$

where

$$
\gamma_{j}=\frac{1}{\mathbf{z}_{j}^{T} \frac{\partial \mathbf{D}\left(s_{j}\right)}{\partial s_{j}} \mathbf{z}_{j}}
$$

From Equations (9) and (10) it can be immediately seen that conventional modal analysis can be extended to general frequency depended damped system in a familiar manner provided the non-linear eigenvalue problem in Eq. (11) can be solved efficiently. This paper is devoted to address this challenging problem.

\section{Non-linear Eigenvalue Problem for Frequency Depended Damped Systems}

The eigenvalue problem associated with a linear system with exponential damping model can be expressed from Eq. (11) as

$$
\left[s_{j}^{2} \mathbf{M}+s_{j} \sum_{k=1}^{n} \frac{\mu_{k}}{s_{j}+\mu_{k}} \mathbf{C}_{k}+\mathbf{K}\right] \mathbf{z}_{j}=\mathbf{0}, \quad \text { for } j=1, \cdots, m .
$$

For systems with viscous damping (i.e, when $\mu_{k} \rightarrow \infty, \forall k$ ) the order of the characteristic polynomial $m=2 N$. For systems with exponential damping model in general $m$ is more than $2 N$, that is $m=2 N+p ; p \geq 0$. Using the state-space approach ${ }^{16}$ it was shown that

$$
p=\sum_{k=1}^{n} \operatorname{rank}\left(\mathbf{C}_{k}\right)
$$

$$
4 \text { of } 17
$$


Therefore, if all of the $\mathbf{C}_{k}$ matrices are of full rank, then $p=n N$. This shows that although the system has $N$ degrees-of-freedom, the number of eigenvalues is more than $2 N$. This is a major difference between a non-viscously damped system and a viscously damped system where the number of eigenvalues is exactly $2 N$, including any multiplicities. When $s_{j}$ appear in complex conjugate pairs, $\mathbf{z}_{j}$ also appear in complex conjugate pairs, and when $s_{j}$ is real $\mathbf{z}_{j}$ is also real. Corresponding to the $2 N$ complex conjugate pairs of eigenvalues, the $N$ eigenvectors together with their complex conjugates are called elastic modes or vibration modes. These modes are related to the $N$ modes of vibration of the structural system. Physically, the assumption of ' $2 N$ complex conjugate pairs of eigenvalues' implies that all the elastic modes are oscillatory in nature, that is, they are sub-critically damped. The modes corresponding to the 'additional' $p$ eigenvalues are called non-viscous modes or overdamped modes. For stable passive systems the non-viscous modes are over-critically damped (i.e., negative real eigenvalues) and not oscillatory in nature. Non-viscous modes, or similar to these, are known by different names in the literature of different subjects, for example, 'wet modes' in the context of ship dynamics ${ }^{25}$ and 'damping modes' in the context of viscoelastic structures. ${ }^{5}$

For the convenience of analytical development, the following four cases are considered:

- single-degree-of-freedom system with single exponential kernel $(N=1, n=1)$

- single-degree-of-freedom system with multiple exponential kernels $(N=1, n>1)$

- multiple-degree-of-freedom system with single exponential kernel $(N>1, n=1)$

- multiple-degree-of-freedom system with multiple exponential kernels $(N>1, n>1)$

In the following sections closed-form approximate expressions of eigenvalues and eigenvectors are derived for the elastic modes and non-viscous modes.

\section{Single-degree-of-freedom System with Single Exponential Kernel}

Computational cost and other relevant issues identified before in the paper do not strictly affect the eigenvalue problem of a single-degree-of-freedom system (SDOF) with exponential damping. The main reason for considering a SDOF system is that in many cases the underlying approximation method can be extended to MDOF systems in a relatively straight-forward manner. For this case when $N=1, n=1$ the eigenvalue equation can be simplified from Eq. (15) as

$$
s^{2} m_{u}+s g(s)+k_{u}=0 \quad \text { where } \quad g(s)=\frac{\mu}{s+\mu} c .
$$

Here we have omitted the subscripts $j$ and $k$ for notational convenience and the matrices appearing in Eq. (15) has been replaced by corresponding scalars. Equation (17) is a third-order polynomial in $s$ and it can be solved exactly in closed-form. A more detailed study on the properties of the exact solutions have been carried out in references. ${ }^{26,27}$ In the next two sections we derive the approximate solutions with the vision that they can be generalized to MDOF systems with minor modifications.

\section{IV.A. Complex-conjugate Solution}

The main motivation of the approximations is that the approximate solution can be 'constructed' from the solution of equivalent viscously damped system. The solution of equivalent viscously damped system can in turn be expressed in terms of the undamped eigensolutions. Combining these together, one can therefore obtain the eigensolutions of frequency-dependent systems by simple 'post-processing' of undamped solutions only. The eigenvalues (appearing in a complex conjugatae pair) of the equivalent viscously damped system is given by ${ }^{28}$

$$
s_{0}=-\zeta_{n} \omega_{n} \pm \mathrm{i} \omega_{n} \sqrt{1-\zeta_{n}^{2}} \approx-\zeta_{n} \omega_{n} \pm \mathrm{i} \omega_{n}
$$

where the undamped natural frequency $\omega_{n}=\sqrt{k_{u} / m_{u}}$ and the viscous damping factor $\zeta_{n}=c / 2 \sqrt{k_{u} m_{u}}$. Viscous damped system is a special case of Eq. (17) when the function $g(s)$ is replaced by $g(s \rightarrow \infty)$. For that case the solution given by Eq. (18) would have been the exact solution of the characteristic equation (17). Since in general this is not the case, the difference between the viscous solution and the true solution of the characteristic equation (17) is essentially arising due to the 'varying' nature of the function $g(s)$. 
The central idea here is that the actual solution of the characteristic equation (17) can be obtained by expanding the solution in a Taylor series around $s_{0}$. The error arising in the resulting solution would then depend on the 'degree of variability' of the function $g(s)$. We assume that the true solution of Eq. (17) can be expressed as

$$
s=s_{0}+\delta
$$

where $\delta$ is a small quantity. Substituting this into the characteristic equation we have

$$
\left(s_{0}+\delta\right)^{2} m_{u}+\left(s_{0}+\delta\right) g\left(s_{0}+\delta\right)+k_{u}=0 .
$$

Expanding $g\left(s_{0}+\delta\right)$ in a Taylor series in $\delta$ around $s_{0}$ and keeping only the first-order terms in $\delta$ we have

$$
\delta^{(1)}=\frac{s_{0}\left(s_{0} m_{u}+g\left(s_{0}\right)\right)+k_{u}}{s_{0}\left(2 m_{u}+g^{\prime}\left(s_{0}\right)\right)+g\left(s_{0}\right)}
$$

where

$$
g\left(s_{0}\right)=\frac{\mu}{s_{0}+\mu} c \quad \text { and } \quad g^{\prime}\left(s_{0}\right)=-\frac{\mu}{\left(s_{0}+\mu\right)^{2}} c .
$$

Here the superscript 1 is used to denote that this is a first-order approximation. Further simplifying, the final solution can be expresses as

$$
s=s_{0}+\delta^{(1)}=s_{0}-\frac{s_{0}\left(s_{0} m_{u}+c \frac{\mu}{\mu+s_{0}}\right)+k_{u}}{s_{0}\left(2 m_{u}-c \frac{\mu}{\left(\mu+s_{0}\right)^{2}}\right)+c \frac{\mu}{\mu+s_{0}}} .
$$

One can improve the accuracy by retaining higher-order terms in $\delta$. Retaining upto second-order terms in $\delta$ in the Taylor expansion of Eq. (20) we have

$$
\delta^{(2)}=\frac{-B-\sqrt{B^{2}-4 A C}}{2 A}
$$

where

$$
\begin{aligned}
A & =\left(m_{u}+\frac{g^{\prime \prime}\left(s_{0}\right)}{2 !} s_{0}+g^{\prime}\left(s_{0}\right)\right) \\
B & =\left(2 m_{u} s_{0}+s_{0} g^{\prime}\left(s_{0}\right)+g\left(s_{0}\right)\right) \\
\text { and } C & =\left(s_{0}^{2} m_{u}+s_{0} g\left(s_{0}\right)+k\right)
\end{aligned}
$$

In the above expressions $g^{\prime}\left(s_{0}\right)$ and $g^{\prime \prime}\left(s_{0}\right)$ are respectively the first and second order derivative of $g(s)$ evaluated at $s=s_{o}$. Our numerical works show that retaining terms higher than the second order results considerably complex expressions and the accuracy gained is not very significant. As a result we have not perused this approach in the rest of the paper. Based on our numerical works we recommend the secondorder expression in Eq. (24) as it gives excellent accuracy and additional computation cost is just marginally higher compared to the first-order approximation. The expressions of the approximate eigenvalue derived here shows that the complex-conjugate solution of the frequency-dependent damped system can be obtained by post-processing of the undamped eigenvalue $\omega_{n}$.

\section{IV.B. Real Solution}

While the complex-conjugate solution can be expected to be close to the solution of the equivalent viscously damped system, no such analogy can be made for the real solution as the equivalent viscously damped system doesn't have one. In order to proceed, we first multiply the characteristic equation $(17)$ by $(s+\mu)$ and rewrite as

$$
\left(s^{2} m_{u}+k_{u}\right)(\mu+s)+s c \mu=0 .
$$

To obtain the initial guess we consider that the damping is small so that $s c \mu \approx 0$. Since $\left(s^{2} m_{u}+k_{u}\right) \neq 0$ as we are considering the real solution only, the first guess is obtained as

$$
\mu+s+0=0 \quad \text { or } \quad s_{0}=-\mu .
$$


We take the first approximation of the real root as

$$
s=s_{0}+\Delta=-\mu+\Delta
$$

and substitute it into the characteristic equation to obtain

$$
\begin{aligned}
\left((-\mu+\Delta)^{2} m_{u}+k_{u}\right)^{2} \Delta+(-\mu+\Delta) c \mu & =0 \\
\text { or } \quad \Delta^{3} m_{u}+\Delta^{2}\left(-\mu m_{u}\right)+\Delta\left(\mu^{2} m_{u}+k_{u}+c \mu\right)-\mu^{2} c & =0 .
\end{aligned}
$$

After neglecting all the terms associated with $\Delta^{n}$ for $n>1$ we have

$$
\Delta \approx \frac{\mu^{2} c}{\mu^{2} m_{u}+k_{u}+\mu c}
$$

Therefore, the real solution is given by

$$
s=s_{0}+\Delta \approx-\mu+\frac{\mu^{2} c}{\mu^{2} m_{u}+k_{u}+\mu c} .
$$

\section{Single-degree-of-freedom System with Multiple Exponential Kernels}

For this case the kernel function $g(s)$ takes the form

$$
g(s)=\sum_{k=1}^{n} \frac{\mu_{k}}{s+\mu_{k}} c_{k}, \quad n>1 .
$$

Using this series, a wide range of damping function can be modelled in the frequency domain. The characteristic function with this type of kernel function can be expressed as

$$
s^{2} m_{u}+s \sum_{k=1}^{n} \frac{\mu_{k}}{s+\mu_{k}} c_{k}+k_{u}=0 .
$$

This a polynomial in $s$ of order $(n+2)$ and therefore, this equation has $(n+2)$ roots. In this section we derive approximate solutions with the view of generalizing them to more realistic MDOF systems.

\section{V.A. Complex-conjugate Solution}

If the system is vibrating, then equation (34) must have a pair of complex conjugate root. The complex conjugate solutions derived in subsection IV.A are for a general function $g(s)$ and therefore are valid for this case also. The solutions are therefore given by Eqs. (19) and (21), where $g(s)$ appearing in these equations need to be replaced by the series in Eq. (33). The necessary details are obvious and consequently omitted here.

\section{V.B. Real Solutions}

There are $n$ number of pure real roots corresponding to $n$ terms in the series in Eq. (33). Multiplying the characteristic equation (34) by the product $\prod_{j}^{n}\left(s+\mu_{j}\right)$ we have

$$
\left(s^{2} m_{u}+k_{u}\right) \prod_{j=1}^{n}\left(s+\mu_{j}\right)+s \sum_{k=1}^{n}\left(\mu_{k} c_{k} \prod_{\substack{j=1 \\ j \neq k}}^{n}\left(\mu_{j}+s\right)\right)=0 .
$$

Like the previous case, we use the approximation

$$
s_{k}=\mu_{k}+\Delta_{k}
$$


Substituting this into the characteristic equation and retaining only the first-order terms in $\Delta_{k}$, after some simplifications we have

$$
\Delta_{k} \approx \frac{c_{k} \mu_{k}^{2} \prod_{\substack{j=1 \\ j \neq k}}^{n}\left(\mu_{j}-\mu_{k}\right)}{\left(\mu_{k}^{2} m+k\right) \prod_{\substack{r=1 \\ n \neq k}}^{n}\left(\mu_{r}-\mu_{k}\right)+\mu_{k}\left[-\mu_{k} c_{k} \sum_{\substack{j=1 \\ j \neq k}}^{n} \prod_{\substack{m=1 \\ m \neq j \\ m \neq k}}^{n}\left(\mu_{m}-\mu_{k}\right)-\sum_{\substack{r=1 \\ r \neq k}}^{n} \mu_{r} c_{r} \prod_{\substack{j=1 \\ j \neq n \\ j \neq k}}^{n}\left(\mu_{j}-\mu_{k}\right)+\prod_{\substack{j=1 \\ j \neq k}}^{n} c_{k}\left(\mu_{j}-\mu_{k}\right)\right]}
$$

for $k=1,2, \cdots, n$.

\section{V.C. Numerical Results}

We consider a single-degree-of-freedom system with eight exponential kernels to investigate the accuracy of the approximate solutions derived in this section. For numerical calculation we assume $m=1 \mathrm{~kg}, k=2 \mathrm{~N} / \mathrm{m}$. It is assumed that all $c_{k}$ are of same value so that $c_{k}=2 \zeta \omega_{n}, \forall k=1,2 . ., 8$. The values of $\mu_{k}$ for $k=1,2 . ., 8$ are selected as $1.9442,1.5231,1.9317,1.7657,1.7454,1.9558,2.0677,1.4973$. The values are generated randomly as $\mu_{k}=0.75\left(1+r_{k}\right) \omega_{n}$, where $r_{k}$ are uniform random numbers between $0-1$. The approximate eigenvalues obtained using the proposed method is compared with the results obtained from exact state-space solution in Table 2, The complex conjugate eigenvalues are obtained very accurately using the proposed

Table 2. Exact and approximate eigenvalues of the SDOF system.

\begin{tabular}{llll}
$\mu$ & $\begin{array}{l}\text { Exact solution } \\
\text { (state-space) }\end{array}$ & $\begin{array}{l}\text { Proposed approxi- } \\
\text { mate solution }\end{array}$ & Percentage error \\
\hline 1.9442 & -1.4649 & -1.5135 & 3.3169 \\
1.5231 & -1.5136 & -1.5185 & 0.3237 \\
1.9317 & -1.7123 & -1.7579 & 2.6643 \\
1.7657 & -1.7576 & -1.7613 & 0.2101 \\
1.7454 & -1.8954 & -1.9253 & 1.5767 \\
1.9558 & -1.9380 & -1.9375 & 0.0282 \\
2.0677 & -1.9517 & -1.9527 & 0.0516 \\
1.4973 & -2.0560 & -2.0592 & 0.1559 \\
Complex Conjugate & $-0.0619 \pm 1.4718 \mathrm{i}$ & $-0.0619 \pm 1.4718 \mathrm{i}$ & $0.0003 \pm 0 \mathrm{i}$ \\
solution & & & \\
\hline
\end{tabular}

approximation. The real eigenvalues are not as accurate as the complex conjugate eigenvalues. However, recall that the motion corresponding to the real eigenvalues are purely dissipative in nature and therefore do not significantly affect the dynamic response of the system. To show this, we have plotted the frequency response function of the system in figure 1. Because the complex conjugate eigenvalues are approximated very well, the frequency response function of the system obtained using the exact and approximate eigenvalues match very well. Again recall that all of the approximate eigenvalues are obtained by post-processing of the undamped eigenvalues only.

\section{Multiple-degree-of-freedom System}

Based on the results for the SDOF system, we derive the complex eigenvalues and eigenvectors for a general $\mathbf{G}(s)$. Complex eigensolutions are also known as elastic eigensolutions as they appear in underdamped elastic systems. For the real eigenvalues two cases namely, when $n=1$ and when $n>1$ are considered for analytical convenience. 


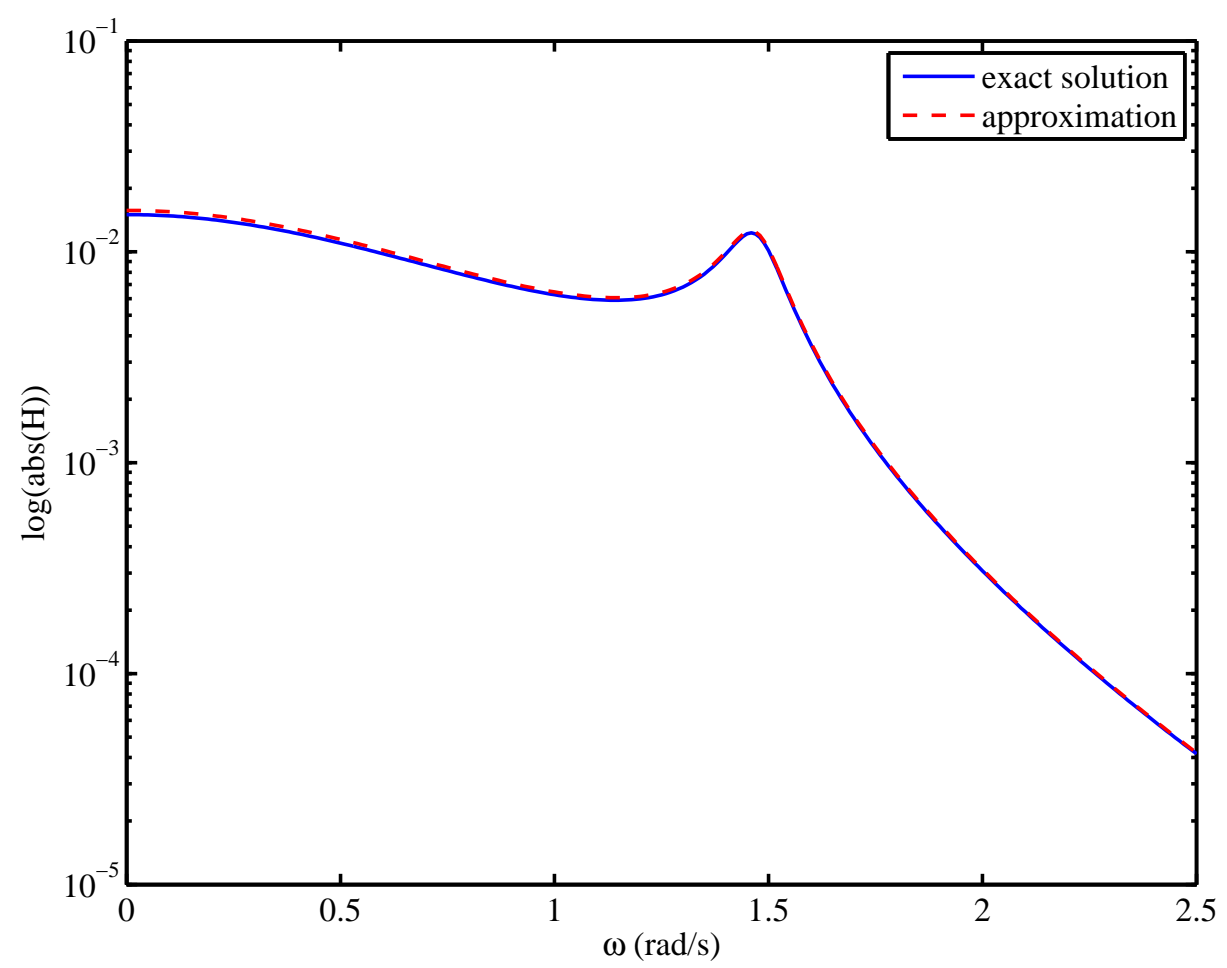

Figure 1. Frequency response function of the SDOF system obtained using exact and approximate eigenvalues.

\section{VI.A. Complex-conjugate Solutions}

The aim of this section is to obtain the complex conjugate eigensolutions using the undamped eigensolutions. The undamped eigenvalue problem of a MDOF system is given by

$$
\mathbf{K} \mathbf{x}_{j}=\omega_{j}^{2} \mathbf{M} \mathbf{x}_{j}
$$

where $\omega_{j}^{2}$ and $\mathbf{x}_{j}$ are the eigenvalues and eigenvectors of the system. The eigenvectors are mass-normalized so that

$$
\begin{aligned}
\mathbf{x}_{l}^{T} \mathbf{M} \mathbf{x}_{j} & =\delta_{l j} \\
\text { and } \quad \mathbf{x}_{l}^{T} \mathbf{K} \mathbf{x}_{j} & =\omega_{j}^{2} \delta_{l j}, \quad \forall l, j=1, \cdots, N
\end{aligned}
$$

where $\delta_{l j}$ is the Kroneker delta function. For distinct undamped eigenvalues $\left(\omega_{l}^{2}\right), \mathbf{x}_{l}, \forall l=1, \cdots, N$, form a complete set of vectors. For this reason, $\mathbf{z}_{j}$ can be expanded as a complex linear combination of $\mathbf{x}_{l}$. Thus, an expansion of the form

$$
\mathbf{z}_{j}=\sum_{l=1}^{N} \alpha_{l}^{(j)} \mathbf{x}_{l}
$$

may be considered. Now, without any loss of generality, we can assume that $\alpha_{j}^{(j)}=1$ (normalization) which leaves us to determine $\alpha_{l}^{(j)}, \forall l \neq j$. Substituting the expansion of $\mathbf{z}_{j}$, from equation (11) one obtains

$$
\sum_{l=1}^{N} s_{j}^{2} \alpha_{l}^{(j)} \mathbf{M} \mathbf{x}_{l}+s_{j} \alpha_{l}^{(j)} \mathbf{G}\left(s_{j}\right) \mathbf{x}_{l}+\alpha_{l}^{(j)} \mathbf{K} \mathbf{x}_{l}=\mathbf{0} .
$$

Premultiplying above equation by $\mathbf{x}_{k}^{T}$ and using the mass-orthogonality property of the undamped eigenvectors one obtains

$$
s_{j}^{2} \alpha_{k}^{(j)}+s_{j} \sum_{l=1}^{N} \alpha_{l}^{(j)} G_{k l}^{\prime}\left(s_{j}\right)+\omega_{k}^{2} \alpha_{k}^{(j)}=0, \quad \forall k=1, \cdots, N
$$

$$
9 \text { of } 17
$$


where $G_{k l}^{\prime}\left(s_{j}\right)=\mathbf{x}_{k}^{T} \mathbf{G}\left(s_{j}\right) \mathbf{x}_{l}$. We consider that the system is lightly non-proportionably damped. For light non-proportionally damped systems, the off-diagonal entries of the modal damping function matrix is small compared to the diagonal entries, that is $G_{k l}^{\prime}\left(s_{j}\right) \leq G_{k k}^{\prime}\left(s_{j}\right), \forall k \neq l, s_{j}$. Considering the $j$-th set of equation (43) and neglecting the second-order terms involving $\alpha_{k}^{(j)}$ and $G_{k l}^{\prime}\left(s_{j}\right), \forall k \neq l$, and also noting that $\alpha_{j}^{(j)}=1$, one obtains

$$
s_{j}^{2}+s_{j} G_{j j}^{\prime}\left(s_{j}\right)+\omega_{j}^{2} \approx 0
$$

This equation is similar to (17) and can be solved in exactly the same way. The modal damping factor $\zeta_{j}$ can be defined as

$$
\zeta_{j}=\frac{G_{j j}^{\prime}(s \rightarrow \infty)}{2 \omega_{j}}=\frac{C_{j j}^{\prime}}{2 \omega_{j}}
$$

where $C_{j j}^{\prime}=\mathbf{x}_{j}^{T} \mathbf{C x}_{j}$ and the matrix $\mathbf{C}$ is defined in Eq. (5).

To obtain the eigenvectors we rewrite Eq. (43) for $j \neq k$ as

$$
\begin{aligned}
& s_{j}^{2} \alpha_{k}^{(j)}+s_{j}\left(G_{k j}^{\prime}\left(s_{j}\right)+\alpha_{k}^{(j)} G_{k k}^{\prime}\left(s_{j}\right)+\sum_{l \neq k \neq j}^{N} \alpha_{l}^{(j)} G_{k l}^{\prime}\left(s_{j}\right)\right)+\omega_{k}^{2} \alpha_{k}^{(j)}=0, \\
& \forall k=1, \cdots, N ; \neq j .
\end{aligned}
$$

These equations can be combined into a matrix form as

$$
\left[\mathbf{P}^{(j)}-\mathbf{Q}^{(j)}\right] \hat{\mathbf{a}}^{(j)}=\mathbf{g}_{\mathbf{u}}{ }^{(j)} .
$$

In the above equation:

$$
\begin{aligned}
& \mathbf{P}^{(j)}=\operatorname{diag}\left[\frac{s_{j}^{2}+s_{j} G_{11}^{\prime}\left(s_{j}\right)+\omega_{1}^{2}}{-s_{j}}, \ldots,\{j-\text { th term deleted }\}, \cdots, \frac{s_{j}^{2}+s_{j} G_{N N}^{\prime}\left(s_{j}\right)+\omega_{N}^{2}}{-s_{j}}\right], \\
& \mathbf{Q}^{(j)}=\left[\begin{array}{cccccc}
0 & G_{12}^{\prime}\left(s_{j}\right) & \ldots & \{j \text {-th term deleted }\} & \ldots & G_{1 N}^{\prime}\left(s_{j}\right) \\
G_{21}^{\prime}\left(s_{j}\right) & 0 & \vdots & \vdots & \vdots & G_{2 N}^{\prime}\left(s_{j}\right) \\
\vdots & \vdots & \vdots & \{j \text {-th term deleted }\} & \vdots & \vdots \\
\vdots & \vdots & \vdots & \vdots & \vdots & \vdots \\
G_{N 1}^{\prime}\left(s_{j}\right) & G_{N 2}^{\prime}\left(s_{j}\right) & \ldots & \{j \text {-th term deleted }\} & \ldots & 0
\end{array}\right] \\
& \mathbf{g}_{\mathbf{u}}{ }^{(j)}=\left\{G_{1 j}^{\prime}\left(s_{j}\right), G_{2 j}^{\prime}\left(s_{j}\right), \cdots,\{j-\text { th term deleted }\}, \cdots, G_{N j}^{\prime}\left(s_{j}\right)\right\}^{T}
\end{aligned}
$$

and

$$
\hat{\mathbf{a}}^{(j)}=\left\{\alpha_{1}^{(j)}, \alpha_{2}^{(j)}, \cdots,,^{\{j-\text { th term deleted }\}}, \cdots, \alpha_{N}^{(j)}\right\}^{T}
$$

is the vector of unknown $\alpha_{k}^{(j)}, \forall k \neq j$. From equation (47), $\hat{\mathbf{a}}^{(j)}$ has to be determined by performing the associated matrix inversion and is achieved by using the Neumann expansion method. Now, using the Neumann expansion we have

$$
\begin{aligned}
\hat{\mathbf{a}}^{(j)} & =\left[\mathbf{I}_{N-1}-\mathbf{P}^{(j)^{-1}} \mathbf{Q}^{(j)}\right]^{-1}\left\{\mathbf{P}^{(j)^{-1}} \mathbf{g}_{\mathbf{u}}{ }^{(j)}\right\} \\
& =\left[\mathbf{I}_{N-1}+\mathbf{R}_{\mathbf{u}}{ }^{(j)}+\mathbf{R}_{\mathbf{u}}^{(j)^{2}}+\mathbf{R}_{\mathbf{u}}{ }^{(j)^{3}}+\cdots\right] \mathbf{a}_{0}^{(j)}
\end{aligned}
$$

where $\mathbf{I}_{N-1}$ is a $(N-1) \times(N-1)$ identity matrix,

$$
\mathbf{R}_{\mathbf{u}}^{(j)}=\mathbf{P}^{(j)^{-1}} \mathbf{Q}^{(j)} \in \mathbb{C}^{(N-1) \times(N-1)}
$$

and

$$
\mathbf{a}_{0}^{(j)}=\mathbf{P}^{(j)^{-1}} \mathbf{g}_{\mathbf{u}}^{(j)} \in \mathbb{C}^{(N-1)} .
$$


Because $\mathbf{P}^{(j)}$ is a diagonal matrix, its inversion can be carried out analytically and subsequently the closedform expressions of $\mathbf{R}_{\mathbf{u}}{ }^{(j)}$ and $\mathbf{a}_{0}^{(j)}$ can be obtained as

$$
\begin{gathered}
R_{u_{k l}}^{(j)}=\frac{-s_{j} G_{k l}^{\prime}\left(s_{j}\right)\left(1-\delta_{k l}\right)}{\omega_{k}^{2}+s_{j}^{2}+s_{j} G_{k k}^{\prime}\left(s_{j}\right)}, \quad \forall k, l \neq j \\
a_{0_{l}}^{(j)}=\frac{-s_{j} G_{l j}^{\prime}\left(s_{j}\right)}{\omega_{l}^{2}+s_{j}^{2}+s_{j} G_{l l}^{\prime}\left(s_{j}\right)}, \quad \forall l \neq j .
\end{gathered}
$$

This makes further calculations involving these quantities simpler. From equation (52), $\hat{\mathbf{a}}^{(j)}$ can be calculated in an efficient way as one can write

$$
\hat{\mathbf{a}}^{(j)}=\mathbf{a}_{0}^{(j)}+\mathbf{a}_{1}^{(j)}+\mathbf{a}_{2}^{(j)}+\cdots+\mathbf{a}_{k}^{(j)}+\cdots
$$

where

$$
\mathbf{a}_{1}^{(j)}=\mathbf{R}_{\mathbf{u}}^{(j)} \mathbf{a}_{0}^{(j)}, \mathbf{a}_{2}^{(j)}=\mathbf{R}_{\mathbf{u}}{ }^{(j)} \mathbf{a}_{1}^{(j)}, \cdots \mathbf{a}_{k}^{(j)}=\mathbf{R}_{\mathbf{u}}{ }^{(j)} \mathbf{a}_{k-1}^{(j)} .
$$

This implies that all the $\mathbf{a}_{k}^{(j)}$ can be obtained using successive matrix-vector multiplications only. Noting that $\hat{\mathbf{a}}^{(j)}$ is the vector of $\alpha_{k}^{(j)}, \forall k \neq j$, substitution of it in equation (41) will give the complex modes. It is easy to see that by taking more terms in the series represented by (57), one can obtain the complex modes quite accurately.

For many engineering problems it is often observed that the damping forces are not very 'large' and that by retaining only a few terms in the series expression (57) will result in an acceptable accuracy. Closed-form approximate expressions for the complex modes can be obtained by retaining one and two terms of these series. Considering only the first term of the series (57) and substituting $\hat{\mathbf{a}}^{(j)}$ in equation (41) we obtain

$$
\mathbf{z}_{j} \approx \mathbf{x}_{j}-\sum_{\substack{k=1 \\ k \neq j}}^{N} \frac{s_{j} G_{k j}^{\prime}\left(s_{j}\right) \mathbf{x}_{k}}{\omega_{k}^{2}+s_{j}^{2}+s_{j} G_{k k}^{\prime}\left(s_{j}\right)}
$$

Now, retaining the first two terms of the series expression (57) and substituting $\hat{\mathbf{a}}^{(j)}$ in equation (41) one obtains

$$
\begin{aligned}
\mathbf{z}_{j} \approx \mathbf{x}_{j} & -\sum_{\substack{k=1 \\
k \neq j}}^{N} \frac{s_{j} G_{k j}^{\prime}\left(s_{j}\right) \mathbf{x}_{k}}{\omega_{k}^{2}+s_{j}^{2}+s_{j} G_{k}^{\prime}\left(s_{j}\right)} \\
& +\sum_{\substack{k=1 \\
k \neq j}}^{N} \sum_{\substack{l=1 \\
l \neq j \neq k}}^{N} \frac{s_{j}^{2} G_{k l}^{\prime}\left(s_{j}\right) G_{l j}^{\prime}\left(s_{j}\right) \mathbf{x}_{k}}{\left(\omega_{k}^{2}+s_{j}^{2}+s_{j} G_{k k}^{\prime}\left(s_{j}\right)\right)\left(\omega_{l}^{2}+s_{j}^{2}+s_{j} G_{l l}^{\prime}\left(s_{j}\right)\right)} .
\end{aligned}
$$

The above equation is second-order approximate expressions of the complex modes of the system. These expressions might be useful whenever we find that the off-diagonal entries of the damping kernel-function matrix in the modal domain are small compared to the diagonal elements.

\section{VI.B. Real Solutions}

Real solutions are obtained using an approach similar to SDOF systems. After neglecting the off-diagonal terms of the modal damping function matrix, the governing characteristic equation for every mode can be expressed by Eq. (44). This equation can be solved for the real eigenvalues. For systems with single exponential kernel, following Eqs. (30)-(32), the real eigenvalues can expressed as

$$
s_{l}=-\mu+\Delta_{l}
$$

where

$$
\Delta_{l} \approx \frac{\mu^{2} C_{l l}^{\prime}}{\mu^{2}+\omega_{l}^{2}+\mu C_{l l}^{\prime}} ; \quad \forall l=1,2, \cdots, N
$$


Assuming all coefficient matrices are of full rank, for systems with $n$ kernels there are in general $n N$ number of purely real eigenvalues. The approximate eigenvalues can be obtained using Eqs. (36) and (37) as

$$
s_{l k}=-\mu_{k}+\Delta_{l k}
$$

where

$$
\Delta_{l k} \approx \frac{C_{k_{l l}} \mu_{k}^{2} \prod_{\substack{j=1 \\ j \neq k}}^{n}\left(\mu_{j}-\mu_{k}\right)}{\left(\mu_{k}^{2}+\omega_{l}^{2}\right) \prod_{\substack{r=1 \\ n \neq k}}^{n}\left(\mu_{r}-\mu_{k}\right)+\mu_{k}\left[-\mu_{k} C_{k_{l l}} \sum_{\substack{j=1 \\ j \neq k}}^{n} \prod_{\substack{m=1 \\ m \neq j \\ m \neq k}}^{n}\left(\mu_{m}-\mu_{k}\right)-\sum_{\substack{r=1 \\ r \neq k}}^{n} \mu_{r} c_{r} \prod_{\substack{j=1 \\ j \neq n \\ j \neq k}}^{n}\left(\mu_{j}-\mu_{k}\right)+\prod_{\substack{j=1 \\ j \neq k}}^{n} C_{k_{l l}}\left(\mu_{j}-\mu_{k}\right)\right]}
$$

and $C_{k_{l l}}=\mathbf{x}_{l}^{T} \mathbf{C}_{k} \mathbf{x}_{l}$.

The eigenvectors can be obtained from equation (11) by fixing any one element of the eigenvectors. Since $\mathbf{D}\left(s_{j}\right) \in \mathbb{R}^{n}$, from equations (11) it is easy to see that $\mathbf{z}_{j} \in \mathbb{R}^{N}$. Partition $\mathbf{z}_{j}$ as

$$
\mathbf{z}_{j}=\left\{\begin{array}{l}
\mathbf{z}_{1 j} \\
\mathbf{z}_{\mathbf{2}}
\end{array}\right\} .
$$

We select $\mathbf{z}_{1 j}=1$ so that $\mathbf{z}_{\mathbf{2}} \in \mathbb{R}^{(N-1)}$ has to be determined from equations (11). Further, partition $\mathbf{D}\left(s_{j}\right)$ as

$$
\mathbf{D}\left(s_{j}\right)=\left[\begin{array}{ll}
\mathbf{D}_{11}\left(s_{j}\right) & \mathbf{D}_{12}\left(s_{j}\right) \\
\mathbf{D}_{21}\left(s_{j}\right) & \mathbf{D}_{\mathbf{2 2}}\left(s_{j}\right)
\end{array}\right]
$$

where $\mathbf{D}_{11}\left(s_{j}\right) \in \mathbb{R}, \mathbf{D}_{\mathbf{1 2}}\left(s_{j}\right) \in \mathbb{R}^{1 \times(N-1)}, \mathbf{D}_{\mathbf{2 1}}\left(s_{j}\right) \in \mathbb{R}^{(N-1) \times 1}$ and $\mathbf{D}_{\mathbf{2 2}}^{(j)} \in \mathbb{R}^{(N-1) \times(N-1)}$. In view of (66) and recalling that $\mathbf{z}_{1 j}=1$, from equation (11) we can have

$$
\begin{aligned}
\mathbf{D}_{\mathbf{2 2}}\left(s_{j}\right) \mathbf{z}_{\mathbf{2}} & =-\mathbf{D}_{\mathbf{2 1}}\left(s_{j}\right) \\
\text { or } \quad \mathbf{z}_{\mathbf{2}} j & =-\left[\mathbf{D}_{\mathbf{2 2}}\left(s_{j}\right)\right]^{-1} \mathbf{D}_{\mathbf{2 1}}\left(s_{j}\right) .
\end{aligned}
$$

It may be noted that determination of the non-viscous modes is computationally more demanding than the elastic modes because inversion of an $(N-1) \times(N-1)$ real matrix is associated with each eigenvector.

\section{VI.C. Numerical Results}

We consider a three degree-of-freedom system to illustrate the proposed method. The mass, stiffness and the damping matrices in the Laplace domain for the problem are considered as:

$$
\begin{gathered}
\mathbf{M}=\left[\begin{array}{ccc}
m_{u} & 0 & 0 \\
0 & m_{u} & 0 \\
0 & 0 & m_{u}
\end{array}\right], \\
\mathbf{K}=\left[\begin{array}{ccc}
2 k_{u} & -k_{u} & 0 \\
-k_{u} & 2 k_{u} & -k_{u} \\
0 & -k_{u} & 2 k_{u}
\end{array}\right]
\end{gathered}
$$

and

$$
\mathbf{G}(s)=\mathbf{C} \sum_{k=1}^{6} \frac{\mu_{k}}{s+\mu_{k}}, \quad \text { where } \quad \mathbf{C}=\left[\begin{array}{ccc}
0.30 & -0.15 & -0.05 \\
-0.15 & 0.30 & -0.15 \\
-0.05 & -0.15 & 0.30
\end{array}\right] \text {. }
$$


Table 3. Exact and approximate eigenvalues of the Three-DOF system.

Exact solution (state- Proposed approxi- Percentage error

space) mate solution

\begin{tabular}{lll}
\hline Real solutions & & \\
-0.9380 & -0.9425 & 0.4797 \\
-1.2995 & -1.3044 & 0.3771 \\
-1.4507 & -1.7413 & 20.0317 \\
-1.5754 & -1.8096 & 14.8661 \\
-1.7405 & -1.5761 & 9.4456 \\
-1.8095 & -1.4507 & 19.8287 \\
-0.6301 & -0.6301 & 0 \\
-1.1276 & -1.1276 & 0 \\
-1.4505 & -1.7096 & 17.8628 \\
-1.5507 & -1.8081 & 16.5990 \\
-1.7096 & -1.5507 & 9.2946 \\
-1.8081 & -1.4505 & 19.7777 \\
-0.6798 & -0.6731 & 0.9856 \\
-1.1295 & -1.1289 & 0.0531 \\
-1.4505 & -1.7085 & 17.7870 \\
-1.5501 & -1.8080 & 16.6376 \\
-1.7086 & -1.5501 & 9.2766 \\
-1.8080 & -1.4505 & 19.7732 \\
Complex Conjugate so- & & \\
lutions & & \\
$-0.4109 \pm 2.6579 \mathrm{i}$ & $-0.4116 \pm 2.6591 \mathrm{i}$ & $0.1704 \pm 0.0451 \mathrm{i}$ \\
$-0.4359 \pm 2.0939 \mathrm{i}$ & $-0.4359 \pm 2.0939 \mathrm{i}$ & $0 \pm 0 \mathrm{i}$ \\
$-0.1674 \pm 0.8523 \mathrm{i}$ & $-0.1649 \pm 0.8528 \mathrm{i}$ & $1.4934 \pm 0.0587 \mathrm{i}$ \\
\hline
\end{tabular}

For the numerical value we consider $m_{u}=1 \mathrm{~kg}, k_{u}=1$. It is assumed that all $\mathbf{C}_{k}$ matrices are same and the values of $\mu_{k}$ for $k=1,2 . ., 6$ are selected as $1.4565,1.0185,1.8214,1.4447,1.6154,1.7919$. The approximate eigenvalues obtained using the proposed method is compared with the results obtained from exact state-space solution in Table 3 . The complex conjugate eigenvalues are obtained very accurately using the proposed approximation. The real eigenvalues are not as accurate as the complex conjugate eigenvalues. However, recall that the motion corresponding to the real eigenvalues are purely dissipative in nature and therefore do not significantly affect the dynamic response of the system.

\section{Conclusions}

Multiple degree-of-freedom linear systems with frequency depended damping kernels is considered. It has been assumed that, in general, the mass and stiffness matrices as well as the matrix of the kernel functions cannot be simultaneously diagonalized by any linear transformation. The analysis is restricted to systems with non-repetitive eigenvalues. The transfer function matrix of the system was derived in terms of the eigenvalues and eigenvectors of the second-order system. Exact closed-form expressions of the response due to arbitrary forcing functions and initial conditions were obtained. The response can be expressed as a sum of two parts, one that arises in usual viscously damped systems and the other that occurs due to non-viscous damping.

The calculation of the eigensolutions of frequency-depended damped systems requires the solution of a non-linear eigenvalue problem. In this paper new methods are developed for such eigenvalue problems. Approximate expressions are derived for the complex and real eigenvalues of the SDOF system with single and multiple exponential kernels. These results are then extended to MDOF systems. These approximations 
allow one to obtain the dynamic response of general frequency-depended damped systems by simple postprocessing of undamped eigensolutions. The accuracy of the proposed approximations were verified using numerical examples. The complex conjugate eigensolutions turn out to be more accurate compared to the real eigensolutions. This is particularly encouraging because complex eigensolutions dominate the dynamic response of linear systems. The method presented offers a reduction in computational effort because neither the first-order formalisms nor the additional dissipation coordinates are employed. This approach might provide further physical insight as only familiar undamped natural frequencies and mode shapes are utilized to obtain the eigensolutions and dynamic response of the system.

\section{Acknowledgements}

BP gratefully acknowledges the support of Swansea University for the award of a Graduate Scholarship in computational engineering. SA gratefully acknowledges the support of the Engineering and Physical Sciences Research Council (EPSRC) through the award of an Advanced Research Fellowship.

\section{A. Derivation of the Frequency Response Function}

The frequency response function matrix of a system is the inverse of the dynamic stiffness matrix:

$$
\mathbf{H}(s)=\mathbf{D}^{-1}(s)=\frac{\operatorname{adj}[\mathbf{D}(s)]}{\operatorname{det}[\mathbf{D}(s)]} .
$$

The poles of $\mathbf{H}(s)$, denoted by $s_{j}$, are the eigenvalues of the system. Because it is assumed that all the $m$ eigenvalues are distinct, each pole is a simple pole. From the residue theorem it is known that any complex function can be expressed in terms of the poles and residues, that is, the transfer function has the form

$$
\mathbf{H}(s)=\sum_{j=1}^{m} \frac{\mathbf{R}_{j}}{s-s_{j}} .
$$

Here

$$
\mathbf{R}_{j}=\underset{s=s_{j}}{\mathrm{res}}[\mathbf{H}(s)] \stackrel{\text { def }}{=} \lim _{s \rightarrow s_{j}}\left(s-s_{j}\right)[\mathbf{H}(s)]
$$

is the residue of the transfer function matrix at the pole $s_{j}$.

It turns out that the eigenvectors of the dynamic stiffness matrix play an important role in determining the residues of the transfer function matrix. For any given $s \in \mathbb{C}$, the eigenvalue problem associated with the dynamic stiffness matrix can be expressed by

$$
\mathbf{D}(s) \phi_{k}(s)=\nu_{k}(s) \phi_{k}(s), \quad \forall k=1, \cdots, N .
$$

In the preceding equation the eigenvalues $\nu_{k}(s) \in \mathbb{C}$ are the roots of the characteristic Eq.

$$
\operatorname{det}\left[\mathbf{D}(s)-\nu(s) \mathbf{I}_{N}\right]=0
$$

and $\phi_{k}(s) \in \mathbb{C}^{N}$ is the $k$-th eigenvector of $\mathbf{D}(s)$. The symbols $\nu_{k}(s)$ and $\phi_{k}(s)$ indicate functional dependence of these quantities on the complex parameter $s$. Such a continuous dependence is expected whenever $\mathbf{D}(s)$ is a sufficiently smooth matrix function of $s$. It should be noted that because $\mathbf{D}(s)$ is an $N \times N$ complex matrix for a fixed $s$, the number of eigenvalues (and consequently the eigenvectors) must be $N$. Further, it can be shown that, for distinct eigenvalues, $\phi_{k}(s)$ also satisfy an orthogonality relationship although $\mathbf{z}_{k}$ do not enjoy any such simple relationship. We normalize $\phi_{k}(s)$ such that

$$
\phi_{j}^{T}(s) \phi_{k}(s)=\delta_{k j}, \quad \forall k, j=1, \cdots, N
$$

In view of the above relationship, from Eq. (74) we have

$$
\phi_{j}^{T}(s) \mathbf{D}(s) \phi_{k}(s)=\nu_{k}(s) \delta_{k j}, \quad \forall k, j=1, \cdots, N
$$

or in the matrix form

$$
\boldsymbol{\Phi}^{T}(s) \mathbf{D}(s) \boldsymbol{\Phi}(s)=\boldsymbol{\nu}(s)
$$

$$
14 \text { of } 17
$$


Here

$$
\begin{aligned}
\boldsymbol{\Phi}(s) & =\left[\boldsymbol{\phi}_{1}(s), \boldsymbol{\phi}_{2}(s), \cdots, \boldsymbol{\phi}_{N}(s)\right] \in \mathbb{C}^{n}, \\
\text { and } \quad \boldsymbol{\nu}(s) & =\operatorname{diag}\left[\nu_{1}(s), \nu_{2}(s), \cdots, \nu_{N}(s)\right] \in \mathbb{C}^{n} .
\end{aligned}
$$

It is possible to establish the relationships between the original eigenvalue problem of the system defined by Eq. (11) and that by Eq. (74). Consider the case when the parameter $s$ approaches any one of the system eigenvalues, say $s_{j}$. Since all the $\nu_{k}(s)$ are assumed to be distinct, for nontrivial eigenvectors, comparing equations (11) and (74) we can conclude that one and only one of the $\nu_{k}(s)$ must be zero when $s \rightarrow s_{j}$. Suppose that the $r$-th eigenvalue of the eigenvalue problem (74) is zero when $s \rightarrow s_{j}$. It is also clear that the eigenvector in (74) corresponding to the $r$-th eigenvalue also approaches the eigenvector in (11) as $s \rightarrow s_{j}$. Thus, when $s=s_{j}$ one has

$$
\nu_{r}\left(s_{j}\right)=0 \quad \text { and } \quad \nu_{k}\left(s_{j}\right) \neq 0, \forall k=1, \cdots, N ; \neq r
$$

and also

$$
\phi_{r}\left(s_{j}\right)=\mathbf{z}_{j} .
$$

These equations completely relate the eigensolutions of (11) with (74). Now, these relationships will be utilized to obtain the residues of the transfer function matrix.

From Eq. (78) one has

$$
\mathbf{D}^{-1}(s)=\boldsymbol{\Phi}(s) \boldsymbol{\nu}^{-1}(s) \boldsymbol{\Phi}^{T}(s) .
$$

Using the expression of the transfer function in Eq. (8) and noting that $\boldsymbol{\nu}(s)$ is a diagonal matrix, we may expand the right-hand side of the above equation to obtain

$$
\mathbf{H}(s)=\mathbf{D}^{-1}(s)=\sum_{k=1}^{N} \frac{\phi_{k}(s) \phi_{k}^{T}(s)}{\nu_{k}(s)} .
$$

Separation of the $r$-th term in the above sum yields

$$
\mathbf{H}(s)=\frac{\boldsymbol{\phi}_{r}(s) \boldsymbol{\phi}_{r}^{T}(s)}{\nu_{r}(s)}+\left[\sum_{\substack{k=1 \\ k \neq r}}^{N} \frac{\phi_{k}(s) \boldsymbol{\phi}_{k}^{T}(s)}{\nu_{k}(s)}\right] .
$$

Clearly, when $s \rightarrow s_{j}$, the second term of the right-hand side of Eq. (85) is analytic because according to Eq. (81) $\nu_{k}\left(s_{j}\right) \neq 0, \forall k=1, \cdots, N ; \neq r$. Now, from Eq. (73) the residue at $s=s_{j}$ may be obtained as

$$
\begin{aligned}
& \mathbf{R}_{j} \stackrel{\text { def }}{=} \lim _{s \rightarrow s_{j}}\left(s-s_{j}\right)\left\{\frac{\phi_{r}(s) \phi_{r}^{T}(s)}{\nu_{r}(s)}+\left[\sum_{\substack{k=1 \\
k \neq r}}^{N} \frac{\phi_{k}(s) \phi_{k}^{T}(s)}{\nu_{k}(s)}\right]\right\} \\
& =\lim _{s \rightarrow s_{j}}\left(s-s_{j}\right) \frac{\phi_{r}(s) \phi_{r}^{T}(s)}{\nu_{r}(s)} \\
& =\frac{\left.\boldsymbol{\phi}_{r}(s) \boldsymbol{\phi}_{r}^{T}(s)\right|_{s=s_{j}}}{\left.\frac{\partial \nu_{r}(s)}{\partial s}\right|_{s=s_{j}}}+\lim _{s \rightarrow s_{j}} \frac{\left(s-s_{j}\right) \frac{\partial}{\partial s}\left[\phi_{k}(s) \boldsymbol{\phi}_{k}^{T}(s)\right]}{\frac{\partial \nu_{r}(s)}{\partial s}} \quad \text { (using l'Hôspital's rule) } \\
& =\frac{\mathbf{z}_{j} \mathbf{z}_{j}^{T}}{\left.\frac{\partial \nu_{r}(s)}{\partial s}\right|_{s=s_{j}}} \quad \text { (by Eq. (82)) } .
\end{aligned}
$$

The denominator in the above expression for the residues, $\left.\frac{\partial \nu_{r}(s)}{\partial s}\right|_{s=s_{j}}$, is still unknown. Now, consider the $r$-th eigenvalue problem associated with the dynamic stiffness matrix. Differentiation of Eq. (74) for $k=r$ with respect to $s$ yields

$$
\frac{\partial \mathbf{D}(s)}{\partial s} \boldsymbol{\phi}_{r}(s)+\mathbf{D}(s) \frac{\partial \boldsymbol{\phi}_{r}(s)}{\partial s}=\frac{\partial \nu_{r}(s)}{\partial s} \boldsymbol{\phi}_{r}(s)+\nu_{r}(s) \frac{\partial \boldsymbol{\phi}_{r}(s)}{\partial s} .
$$


Premultiplying the above equation by $\phi_{r}^{T}(s)$ and rearranging one obtains

$$
\boldsymbol{\phi}_{r}^{T}(s) \frac{\partial \mathbf{D}(s)}{\partial s} \boldsymbol{\phi}_{r}(s)+\left[\boldsymbol{\phi}_{r}^{T}(s) \mathbf{D}(s)-\boldsymbol{\phi}_{r}^{T}(s) \nu_{r}(s)\right] \frac{\partial \boldsymbol{\phi}_{r}(s)}{\partial s}=\boldsymbol{\phi}_{r}^{T}(s) \frac{\partial \nu_{r}(s)}{\partial s} \boldsymbol{\phi}_{r}(s) .
$$

Taking transpose of Eq. (74) it follows that the second term of the left-hand side of the above equation is zero. Using the normalizing condition in (76) and letting $s \rightarrow s_{j}$, from Eq. (88) we have

$$
\left.\frac{\partial \nu_{r}(s)}{\partial s}\right|_{s=s_{j}}=\left.\mathbf{z}_{j}^{T} \frac{\partial \mathbf{D}(s)}{\partial s}\right|_{s=s_{j}} \mathbf{z}_{j}=\mathbf{z}_{j}^{T} \frac{\partial \mathbf{D}\left(s_{j}\right)}{\partial s_{j}} \mathbf{z}_{j} .
$$

The term $\frac{\partial \mathbf{D}\left(s_{j}\right)}{\partial s_{j}}$ can be obtained by differentiating Eq. (7) as

$$
\frac{\partial \mathbf{D}\left(s_{j}\right)}{\partial s_{j}}=2 s_{j} \mathbf{M}+\mathbf{G}\left(s_{j}\right)+s_{j} \frac{\partial \mathbf{G}\left(s_{j}\right)}{\partial s_{j}} .
$$

Using (86) and (89) one finally obtains the residue as

$$
\mathbf{R}_{j}=\frac{\mathbf{z}_{j} \mathbf{z}_{j}^{T}}{\mathbf{z}_{j}^{T} \frac{\partial \mathbf{D}\left(s_{j}\right)}{\partial s_{j}} \mathbf{z}_{j}} .
$$

The above equation completely relates the transfer function residues to the eigenvalues and eigenvectors of the system. Recalling that, among the $m$ eigenvalues $2 N$ appear in complex conjugate pairs, the transfer function matrix may be obtained as

$$
\mathbf{H}(\mathrm{i} \omega)=\sum_{j=1}^{N}\left[\frac{\gamma_{j} \mathbf{z}_{j} \mathbf{z}_{j}^{T}}{\mathrm{i} \omega-s_{j}}+\frac{\gamma_{j}^{*} \mathbf{z}_{j}^{*} \mathbf{z}_{j}^{*^{T}}}{\mathrm{i} \omega-s_{j}^{*}}\right]+\sum_{j=2 N+1}^{m} \frac{\gamma_{j} \mathbf{z}_{j} \mathbf{z}_{j}^{T}}{\mathrm{i} \omega-s_{j}},
$$

where

$$
\gamma_{j}=\frac{1}{\mathbf{z}_{j}^{T} \frac{\partial \mathbf{D}\left(s_{j}\right)}{\partial s_{j}} \mathbf{z}_{j}} .
$$

The transfer function matrix has two parts, the first part is due to the elastic modes, and the second part is due to the non-viscous modes.

\section{References}

${ }^{1}$ Biot, M. A., "Linear thermodynamics and the mechanics of solids," Proceedings of the Third U. S. National Congress on Applied Mechanics, ASME, New York, 1958, pp. 1-18.

${ }^{2}$ Biot, M. A., "Variational principles in irreversible thermodynamics with application to viscoelasticity," Physical Review, Vol. 97, No. 6, 1955, pp. 1463-1469.

${ }^{3}$ Bagley, R. L. and Torvik, P. J., "Fractional calculus-a different approach to the analysis of viscoelastically damped structures," AIAA Journal, Vol. 21, No. 5, May 1983, pp. 741-748.

${ }^{4}$ Golla, D. F. and Hughes, P. C., "Dynamics of viscoelastic structures - a time domain finite element formulation," Transactions of ASME, Journal of Applied Mechanics, Vol. 52, December 1985, pp. 897-906.

${ }^{5}$ McTavish, D. J. and Hughes, P. C., "Modeling of linear viscoelastic space structures," Transactions of ASME, Journal of Vibration and Acoustics, Vol. 115, January 1993, pp. 103-110.

${ }^{6}$ Lesieutre, G. A. and Mingori, D. L., "Finite element modeling of frequency-dependent material properties using augmented thermodynamic fields," AIAA Journal of Guidance, Control and Dynamics, Vol. 13, 1990, pp. 1040-1050.

${ }^{7}$ Adhikari, S., Energy Dissipation in Vibrating Structures, Master's thesis, Cambridge University Engineering Department, Cambridge, UK, May 1998, First Year Report.

${ }^{8}$ Adhikari, S. and Woodhouse, J., "Identification of damping: part 1, viscous damping," Journal of Sound and Vibration, Vol. 243, No. 1, May 2001, pp. 43-61.

${ }^{9}$ Gaul, L., Klein, P., and Kemple, S., "Damping description involving fractional operators," Mechanical Systems and Signal Processing, Vol. 5, No. 2, 1991, pp. 81-88.

${ }^{10}$ Ruge, P. and Wagner, N., "Time-domain solutions for vibration systems with fading memory," Proceedings of the European Conference on Computational Mechanics (ECCM-99), edited by W. Wunderlich, München, Germany, 1999.

${ }^{11}$ Wagner, N., "Ein direktes Verfahren zur numerischen Lösung von Schwingungssystemen mit nachlassendem Gedächtnis," Zeitschrift für Angewandte Mathematik und Mechanik (ZAMM), Vol. 81, 2001, pp. S 327-328.

${ }^{12}$ Cremer, L. and Heckl, M., Structure-Borne Sound, Springer-Verlag Berlin, Germany, 2nd ed., 1973, Translated by E. E. Ungar.

${ }^{13}$ Lesieutre, G. A. and Bianchini, E., "Time-domain modeling of linear viscoelasticy using anelastic displacement fields," Transactions of ASME, Journal of Vibration and Acoustics, Vol. 117, No. 4, October 1995, pp. 425-430. 
${ }^{14}$ Muravyov, A. and Hutton, S. G., "Closed-form solutions and the eigenvalue problem for vibration of discrete viscoelastic systems," Transactions of ASME, Journal of Applied Mechanics, Vol. 64, September 1997, pp. 684-691.

${ }^{15}$ Muravyov, A., "Forced vibration responses of a viscoelastic structure," Journal of Sound and Vibration, Vol. 218, No. 5, December 1998, pp. 892-907.

${ }^{16}$ Wagner, N. and Adhikari, S., "Symmetric state-space formulation for a class of non-viscously damped systems," AIAA Journal, Vol. 41, No. 5, 2003, pp. 951-956.

${ }^{17}$ Adhikari, S. and Wagner, N., "Analysis of asymmetric non-viscously damped linear dynamic systems," Transactions of ASME, Journal of Applied Mechanics, Vol. 70, No. 6, December 2003, pp. 885-893.

${ }^{18}$ Woodhouse, J., "Linear damping models for structural vibration," Journal of Sound and Vibration, Vol. 215, No. 3, 1998, pp. $547-569$.

${ }_{19}$ Adhikari, S., "Dynamics of non-viscously damped linear systems," ASCE Journal of Engineering Mechanics, Vol. 128, No. 3, March 2002, pp. 328-339.

${ }^{20}$ Adhikari, S. and Wagner, N., "Direct time-domain approach for exponentially damped systems," Computer and Structures, Vol. 82, No. 29-30, November 2004, pp. 2453-2461.

${ }^{21}$ Adhikari, S., "Classical normal modes in non-viscously damped linear systems," AIAA Journal, Vol. 39, No. 5, May 2001 , pp. 978-980.

${ }^{22}$ Adhikari, S., "Optimal complex modes and an index of damping non-proportionality," Mechanical System and Signal Processing, Vol. 18, No. 1, January 2004, pp. 1-27.

${ }^{23}$ Adhikari, S. and Woodhouse, J., "Identification of damping: part 2, non-viscous damping," Journal of Sound and Vibration, Vol. 243, No. 1, May 2001, pp. 63-88.

${ }^{24}$ Adhikari, S. and Woodhouse, J., "Identification of damping: part 3, symmetry-preserving method," Journal of Sound and Vibration, Vol. 251, No. 3, March 2002, pp. 477-490.

${ }^{25}$ Bishop, R. E. D. and Price, W. G., "An investigation into the linear theory of ship response to waves," Journal of Sound and Vibration, Vol. 62, No. 3, 1979, pp. 353-363.

${ }^{26}$ Adhikari, S., "Qualitative dynamic characteristics of a non-viscously damped oscillator," Proceedings of the Royal Society of London, Series- A, Vol. 461, No. 2059, July 2005, pp. 2269-2288.

${ }^{27}$ Adhikari, S., "Dynamic response characteristics of a non-viscously damped oscillator," Transactions of ASME, Journal of Applied Mechanics, Vol. 75, No. 1, January 2007, pp. 011003:1-12.

${ }^{28}$ Meirovitch, L., Analytical Methods in Vibrations, Macmillan Publishing Co., Inc., New York, 1967.

${ }^{29}$ Friswell, M. I., Inman, D. J., and Lam, M. J., "On the realisation of GHM Models in viscoelasticity," Journal of Intelligent Material Systems and Structures, Vol. 8, No. 11, November 1997, pp. 986-993. 